\title{
How Robust Are the Policy Conclusions of the St. Louis Equation?: Some Further Evidence
}

\author{
Dallas S. Batten and Daniel L. Thornton
}

$\mathrm{N}$ a previous issue of this Review, we provided some evidence that the policy conclusions of the St. Louis equation are robust with respect to both the specification of its lag structure and the imposition of polyno. mial restrictions: monetary policy has a significant long-run effect on aggregate income, while fiscal policy does not. "This result is important because it provides evidence that these policy conclusions are not dependent on the equation's econometric specification, a subject of continued debate since the equation first appeared. This conclusion, however, was based on the use of only one technique - - developed by Pagano and Hartley 1981$)$ - - for selecting the appropriate lag structure and polynomial degree, Consequently, the general sensitivity of the policy conclusions to the specification of lag lengths and polynomial degrees remains an issue.

The purpose of this article is to use various model selection criteria to investigate the impact of model specification on the policy conclusions drawn from the St. Louis equation. ${ }^{2}$ The evidence presented here

Dallas S. Batten and Daniel L. Thornton are senior economists at the Federal Reserve Bank of St. Lou's. Sarah R. Driver provided research assistance.

'See Batten and Thornton (1983).

2Since there has been an increased interest in techniques for specifying lag lengths of finite distributed lag models, our results, although data and model specficic, should provide an experiential starting point for those interested in using these procedures. demonstrates that these conclusions are extremely robust with respect to changes in either the lag structure or the polynomial restrictions. Thus, arguments that the general policy conclusions of the St. Louts equation are dependent upon an ad hoc econometric specification are without merit.

\section{THE PRODLEM OF MODEL SPECIFICATION}

To investigate the appropriate lag lengths for the St. Louis equation, we employ the growth rate specification, presented as equation 1 in table 1 . The dots over each variable represent quarter-to-quarter annual rates of change, and $Y, M$ and $G$ are nominal $G N P$, money the $\mathrm{M} 1$ definition and high-employment govemment expenditures, respectively.

The first problem in estimating the St. Louis equation, or for that matter, any finite distributed lag model, is to specify the order of the distributed lags $(1, \mathrm{~K}$. Model selection criteria ypicaly trade off the bias associated with specifying too short a lag lor too low a polynomial degree against the inefficiency associated with selecting too long a lag lor too high a polynomial degreel. In general if either the lag is too long or the polynomial degree too high, the estimates will be unbiased but inefficient. If either the lag is too short or the polynomial degree too low, the estimates will be biased but efficient. Furthermore, since the St. Louis equation 


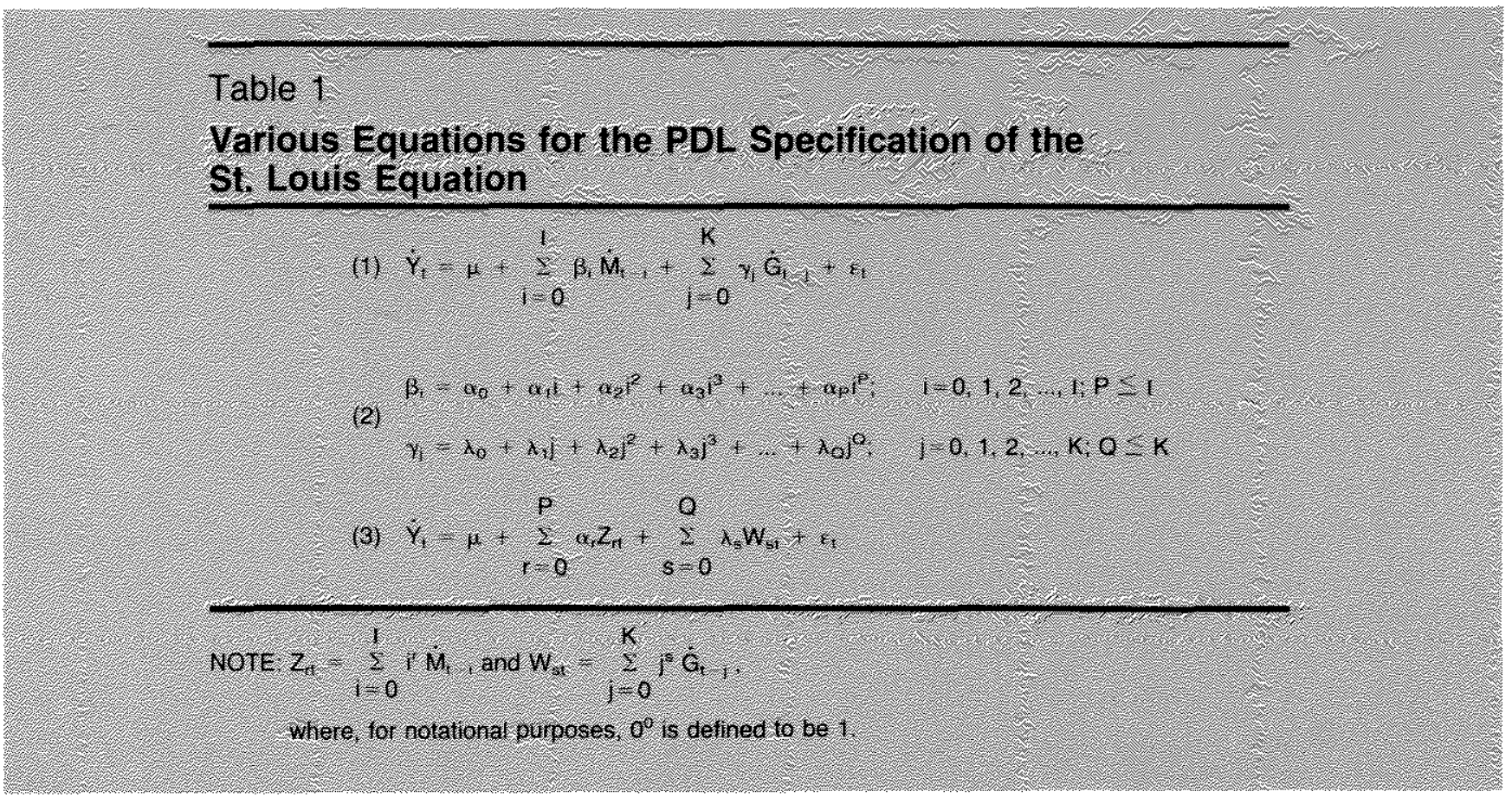

has two distributed lag variables, the resulting estimates will be biased and may be inefficient if one lag is too long and the other too short, ${ }^{3}$

Because different criteria give different weights to this bias/efficiency trade-off, they may select different lag structures and polynomial degrees). In the context of the St. Louis equation, this means that different policy conclusions may be obtained simply because different weights are used for the bias/efficiency tradeoff. In particular, the conclusion that fiscal policy is ineffective in the long run may result largely from the lack of efficiency of the estimator. In order to investigate this issue, we examine the general conclusions concerning monetary and fiscal policy effectiveness in models selected by six different model selection criteria. These criteria were chosen either because they are among the most commonly suggested or because they represent a definite ordering of the bias/efficiency trade-off.

\footnotetext{
${ }^{3}$ The actual conditions are somewhat more complicated than is indicated here. Let $q$ and $x^{*}$ denote the assumed and correct lag length and $p$ and $\mathrm{p}^{*}$ denote the assumed and correct degree of polynomial, respectively. Estimates of the parameter vector will be biased if (a) $q=q^{*}$ and $p<p^{*}$, (b) $q<q^{*}$ and $p=p^{*}$, or (c) $q>q^{*}, p=p^{*}$ and $q-q^{\star}>p^{\star}$. In the instance when $q-q^{\star} \leq p^{\star}$, the polynomial distributed lag estimates may be biased, but need not be. That is, there are restrictions that may or may not be satisfied by the data. Furthermore. PDL estimators will be inefficient if $q=q^{*}$ and $p>p^{*}$. See Trivedi and Pagan (1979).
}

\section{LAG-LENGTH SELECTION}

The criteria employed here are: Pagano and Hartley's t-test (PH), Mallows' (1973) Cp-statistic, Akaike's (1970) Final Prediction Error (FPE), Geweke and Meese's (1981) Bayesian Estimation Criterion (BEC), Schwarz' (1978) Bayesian Information Criterion (SBIC) and the standard F-test. ISee the appendix for a brief description of these criteria.

Each of the six alternative criteria for determining the appropriate lag lengths is used to select the lag lengths $\{\mathrm{I}, \mathrm{K}$ ) for money and government expenditures growth. To assess the sensitivity of the various techniques to the selection of the maximum lag length $\{\mathrm{L}$, three values of $L(8,12$ and 16$)$ are specified initially for each variable.

\section{Empirical Results of Lag-Length Selection}

The $\mathrm{St}$. Louis equation is estimated over the period 11/1962 to III/1982. The results reported in table 2 show

\footnotetext{
${ }^{4}$ While it is unclear how the various criteria will select lags in the general case, it is possible to order the selection when only two altenative lag specifications, $p$ and $p+q$, are considered. The criterion woutd pick $p$, using an F-test, in the following way: $C p$ if $F<2$; $F P E$ if $F<2 T /(T+p+1)$; SBIC if $F<1+\left[(T+p+1)\left(T^{q T}-1\right) / q\right]$; $B E C$ if $F<1+[(T-p-1) \ln T /(T-p-q-1)]$, where $T$ is the sample size.

${ }^{5}$ The sample period is chosen to contorm to that employed in Batten and Thornton.
} 


\section{Table 2}

\section{Lag Lengths Selected by Various Model Selection Criteria}

\begin{tabular}{|c|c|c|c|c|c|c|}
\hline \multirow{3}{*}{ Gricton } & \multicolumn{6}{|c|}{ Marinum tag lengll (U) } \\
\hline & \multicolumn{2}{|c|}{8} & \multicolumn{2}{|c|}{12} & \multicolumn{2}{|c|}{16} \\
\hline & 1 & 6 & M & 6 & n & 6 \\
\hline H. & 5 & 6 & 10 & 9 & 10 & 9 \\
\hline 6 & 5 & 2 & 5 & 2 & 6 & 2 \\
\hline Fe & 5 & 2 & 10 & 9 & 10 & 9 \\
\hline bete & $\sqrt{10}$ & (1) & 1 & 10 & 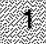 & 0 \\
\hline 816 & 2 & 0 & 2 & (6. & 2 & 0 \\
\hline fros: & X & 8 & 10 & 6 & 1 & 0 \\
\hline
\end{tabular}

that the chosen lag lengths differ by criterion and, to a lesser extent, by the maximum lag length specified. For example, when the maximum lag length was eight, the PH criterion selected the lag on money growth (1) to be five and the lag on govermment expenditure growth ( $\mathrm{K}$ ) to be zero." Both the FPE and Cp criteria choose the same lag on $\dot{M}$ but a slightly longer lag on $\dot{G}$. When the maximum lag is increased to 12 and 16 , both the FPE and PH criteria select longer lags on $\dot{M}$ and $\dot{G}$ if $=10$ and $K=9$. The $\mathrm{Cp}$ statistic, however, is unaffected by changing the maximum lag length. The Bayesian criteria also are unaffected by the choice of the maximum lag length; however, they select lags that are extremely short. Perhaps these criteria give too much weight to efficiency in the bias/efficiency trade-off.

The F-test appears to be the most sensitive to the choice of the maximum lag length. It tends to indicate shorter lags whenever the last significant lag coefficient is followed by a number of insignificant ones. The insignificant coefficients tend to dilute the discriminating power of the $\mathrm{F}$-test. Thus, it chooses a much shorter lag when $L$ is increased from 12 to 16 . This is to be expected, however, given the general nature of this test.

\footnotetext{
${ }^{6}$ Actually, the PH tratio for the second lag of $\dot{G}$ is 1.91 when the tag on $\dot{M}$ is tive. Thus, the $P H$ technique nearly selects the same lag structure (five on $M$ and two on $G$ ) as does the FPE criterion.

'The Bayesian criteria are designed to be asymptotically efficient in that they select the correct lag length in large samples (see Geweke and Meese for details). If appears, however, that they give this property too much weight in small samples and select lags (and polynomial degrees) that are too short. In a Monte Carto experiment. Geweke and Meese found that the probability of underitting is about 50 percent even with a sample size of 50 .
}

\section{Table 3}

\section{Tests of Policy Effectiveness for Various Distributed Lag Models}

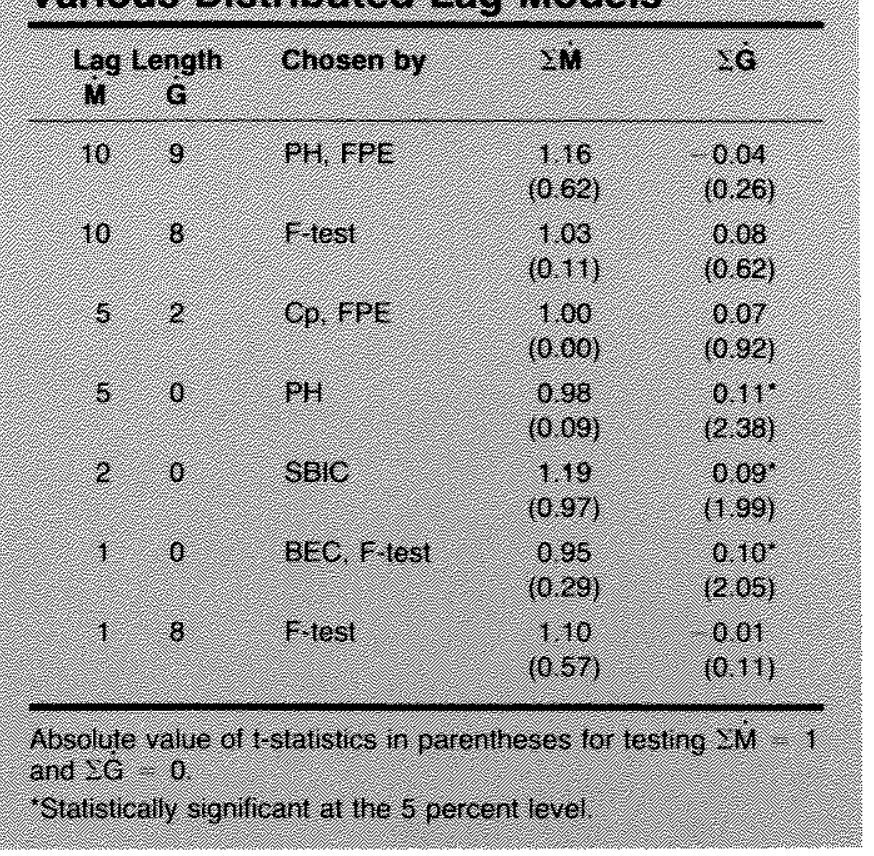

\section{Policy Effectiveness and the Lag Structure}

To test for the long-run effectiveness of monetary and fiscal policies, simple t-tests of the sums of the distributed lag weights are performed. The results of these tests, for the lag structures reported in table 2 , are presented in table 3 . The summed effects of money growth on nominal income growth range from 0.95 to 1.19, and the hypothesis that there is a one-to-one relationship between money growth and growth in nominal income in the long run cannot be rejected for any of the lag structures.

The summed effects of government expenditures on nominal income range from -0.04 to 0.11 and, in contrast to the estimated impacts of changes in money growth, are not significantly different from zero in every instance when there is a lagged effect of $\dot{G}$. This suggests that there is no long-run effect of $\dot{G}$ on nominal income growth. In the three models where only contemporaneous $\dot{\mathrm{G}}$ is included, however, its coef-

Furthermore, for this analysis, the equation is constrained to conlain both variables. That is, the possibility that one of the criteria can select a model in which either $\dot{M}$ or $\dot{G}$ is excluded completely is precluded. If this constraint is removed, however, both Bayesian criteria indicate that not even contemporaneous $\dot{G}$ should be included in the equation. 
ficient is always significant, a result independent of the lag on $\dot{M}^{*}$ This suggests that high-employment govemment expendifures may have an immediate and permanent impact on nominal output. When the models with "long" lags on both variables $(\dot{M}$ and $\dot{G}$ ) are tested against a model with a "long" lag on $\dot{M}$ and no lag on G via a likelihood ratio test, however, the latter model is rejected at the 5 percent significance level. ${ }^{9}$ That is, the model with long lags on both variables is preferred. In the preferred specification, $G$ has a significant short-run effect, but no long-run effect on nominal output.

The above results suggest that the long-run policy implications of the St. Louis equation are relatively insensitive to the lag specification and, hence, to the relative weighting of the bias/efficiency trade-off. Only in the models chosen by the Bayesian criteria does government spending have a pemanent effect on income. The data suggest, however, that longer lag specifications are preferable over the short ones chosen by the Bayesian criteria. Consequently, it appears that these criteria give too much weight to efficiency in the bias efficiency trade-off.

It should be noted that, even though the long-run (equilibrium) properties of the equation are quite robust with respect to the lag structure, the shortrun dynamics differ considerably, especially for a change in money growth. In particular, the short-run impact of a change in money growth is considerably larger, and lasts longer, in the models with relatively long lags on money growth than in the shorter lag specifications.

\section{POL NOMMAL-DEGREE SELECTITN}

The problem of polynomial-degree selection is completely analogous to that of lag-length selection. To see this, we note that the polynomial distributed lag (PDL) estimation technique assumes that the regression coefficients on $\dot{M}$ and $\dot{G}$ (i.e., the $\beta$ s and $\gamma s$ fall on polynomials of degrees $\mathrm{P}$ and $\mathrm{Q}$, respectively, where $\mathrm{P} \leq \mathrm{I}$ and $\mathrm{Q} \leq \mathrm{K}$. These assumptions are given by the equations $(2)$ in table 1 . Given the lag lengths, $I$ and $K$, the equations in $(2)$ can be combined with (1) to obtain the PDL equation (3). Thus, selecting the polynomial degree amounts to choosing orders $(P, Q)$ of equation

Estimates of the equations that include only contemporaneous $\dot{G}$ and lags of $\dot{M}$ from 1 to 10 are not qualitatively different from those reported in table 3 .

"When models with 10 lags on $\dot{M}$ and 9 or 8 lags of $\dot{G}$ are compared with a model with to lags on $\dot{M}$ and only contemporaneous

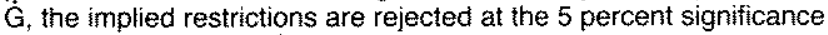
fevel. The calculated $x^{2}$ statistics ( 5 percent critical values) are 24.39 $\left(x^{2}(9)=16.9\right)$ and $17.28\left(x^{2}(8)=15.5\right)$, respectively.
(3). As with the specification of lag lengths, we must specify the maximum polynomial degree that will be considered initially. In this instance, however, the choice is not arbitrary because the polynomial degree cannot be larger than the lag length of the model we are considering.

The application of the above procedure to all of the models in the previous section would be tedious since seven different lag structures were selected by the various criteria for different maximum lag lengths. Thus, to simplify choosing the polynomial degree, a "best" lag structure is chosen. To do this, each lag structure in table 2 is tested against the others and against arbitrarily chosen lags of four, six and twelve on both $\dot{M}$ and $\mathrm{G}$ using a likelihood fatio test. The resulting $x^{2}$ statistics are reported in table 4 . Because some of the lag structures reported in table 2 differ only slightly from each other, the results of all the tests are not reported.

These results indicate that the model with 10 lags on $\dot{M}$ and 9 lags on $\dot{G}$ does well felative to all the others. For example, when this model is tested against the arbitrary model with six lags on both variables, the null hypothesis that the additional four lags on $\dot{M}$ and the additional three lags on $\dot{G}$ are all zero is rejected at the 5 percent significance level. This is also true of the other "short" lag models. Furthermore, when this model is compared with one with twelve lags on both variables, the null hypothesis that the additional two lags on $\dot{\mathrm{M}}$ and the additional three lags on $\dot{G}$ are all zero cannot be rejected. Indeed, only the longer lags chosen by the $P H$ and FPE criteria cannot be rejected relative to all of the other specifcations. Finally, it is interesting to note that the extremely short lags of the Bayesian criteria are generally rejected relative to the longer lag specifications. While no amount of testing can be conclusive, these results suggest that the longer lag structures selected by the PH and FPE criteria are the most appropriate. Consequently, the results of polynomial-degree selection, which are reported below, are based on a model with 10 lags on $\dot{M}$ and 9 on $\dot{G}$.

\section{Empirical Results of Polynomial-Degree Selection}

The same six criteria used to determine the lag length were applied to the selection of the polynomial degree. ${ }^{10}$ The results are presented in table $5 .{ }^{11}$ These

\footnotetext{
${ }^{10}$ Because of their spurious nature, the endpoint constraints are not imposed; see Thornton and Batten (1984).

${ }^{11}$ The polynomial degrees chosen by the PH criterion here differ from those reported in Batten and Thornton. In that article we attempted to account for the preliminary test problem.
} 


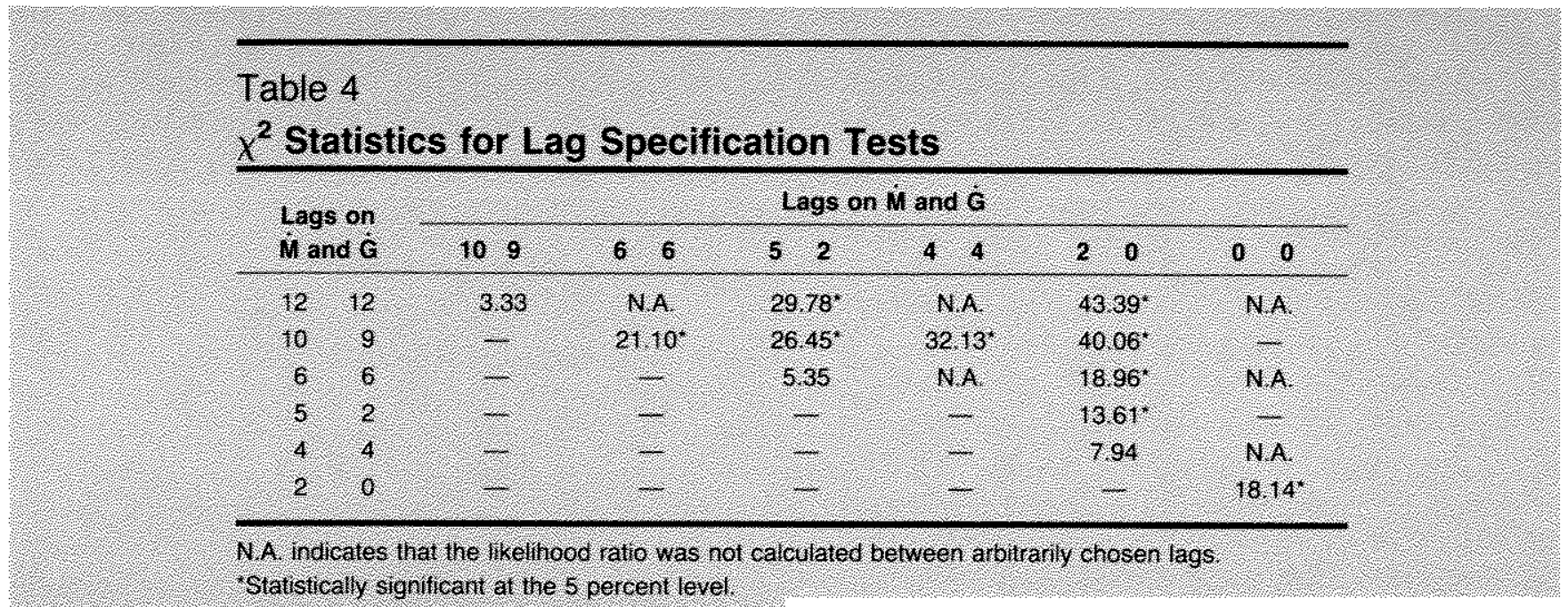

\section{Table 5}

\section{Polynomial Degrees Selected by Various Model Selection Criteria}

\begin{tabular}{|c|c|c|}
\hline & \multicolumn{2}{|c|}{ Polynomial degree } \\
\hline Orterion & N & G \\
\hline PH & 6 & 7 \\
\hline 60 & 6 & 6 \\
\hline pe & 6 & 8 \\
\hline EEO & 10 & 6 \\
\hline SEIC & 1 & 0 \\
\hline 16st & 6 & 3 \\
\hline
\end{tabular}

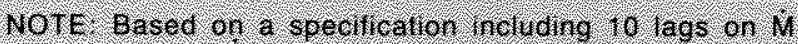
and 9 on $\mathrm{G}$

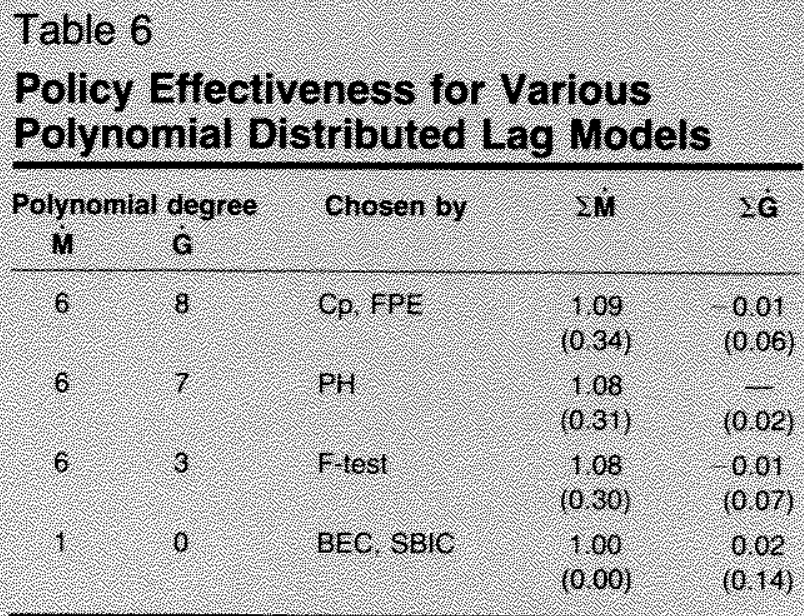

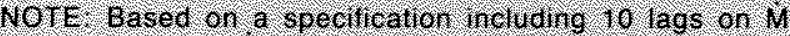
and 9 on 6 . Absclute value or 1 stalistics in paren. theses for testing $2 \mathrm{~N}$, 1 and \& $\mathrm{G}, 0$

2. ndicates less nan 005 in absolute value. results are similar to those obtained in the lag-length selection in that the FPE and PH criteria land in this instance, Mallows' Cpl select relatively high polynomial degrees, while the Bayesian criteria select extremely low degree polynomials. The Bayesian results suggest that estimates of the 11 coefficients on $\dot{M}$ (contemporaneous plus the 10 distributed lags) and the 10 on $G$ can be obtained by estimating only 2 polynomial coefficients on $\dot{M}$ and only 1 on $\dot{G}$. Indeed, when the polynomial restrictions implied by the model selected by these criteria are tested, they are rejected at the 5 percent significance level. On the other hand, when the implied polynomial restrictions of the FPE. and PH determined specifications are tested, they cannot be rejected. ${ }^{12}$

\section{Policy Efectiveness and the Polynomial Degree}

Tests of the policy implications from these PDL models are presented in table 6 . Again, the results confirm the robustness of the policy implications of the $\mathrm{St}$. Louis equation. The summed effects of money growth on nominal income growth differ only slightly from those reported in table 3 and range from 1.00 to 1.09 Furthermore, a test of the hypothesis that there is a one to-one relationship between the growth rate in money and nominal income growt hannot be rejected at the 5 percent significance level for the various sets of polynomial restrictions.

\footnotetext{
${ }^{12}$ The $x^{2}$ statistic for testing the polynomial restrictions selected by the Bayesian criteria is 52.64, compared with a critical value of $\chi^{2}(18)=$ 28.9. For the FPE, PH and F-test selected PDL models, the $x^{2}$ statistics ( 5 percent critical values) are $8.62\left(x^{2}(5)=11.1\right), 11.19$ $\left(x^{2}(6)=12.6\right)$ and $23.21\left(x^{2}(10)=18.3\right)$, respectively.
} 
Also, the summed coefficients on $\dot{G}$ are nearly zero and the hypothesis that they are equal to zero cannot be rejected at the 5 percent significance level. Thus, the policy implications of the St. Louis equation also appear to be unaffected by the choice of polynomial degree.

\section{CONCLUSIONS}

This paper has investigated the robustness of the policy conclusions of the St. Louis equation with respect to its polynomial distributed lag specification. Six alternative model specification criteria have been used to identify lag lengths and polynomial degrees, and tests of policy effectiveness have been performed on each of these specifications.

In each case, the hypothesis that a 1 percentage point increase in money growth leads ultimately to a 1 percentage point increase in the rate of growth of nominal GNP cannot be rejected at conventional levels of statistical significance. Alternatively, highemployment government spending has a permanent impact on the rate of growth of nominal GNP only when contemporaneous government spending growth alone is included with the distributed lag of money growth in the model. This specification, however, is consistently rejected when tested against higher order specifications. Consequently, the general conclusion from this study is that in the long run, monetary policy is effective and fiscal policy is ineffective in influencing the growth of GNP. This result is almost totally insensitive to alternative lag structures or polynomial specifications,

\section{PERERENCES}

Akaike, Hirotugu. "Statistical Predictor Identification," Annals of The Institute of Statistical Mathematics (no. 2, 1970), pp. 203-17.

Batten, Dallas S., and Daniel L. Thornton. "Polynomial Distributed Lags and the Estimation of the St. Louis Equation," this Review (April 1983), pp. 13-25.

Geweke, John, and Richard Meese. "Estimating Regression Models of Finite but Unknown Order," International Economic Review (February 1981), pp. 55-70.

Hsiao, Cheng. "Autoregressive Modelting and Money-Income Causality Detection," Journal of Monetany Economics (January 1981), pp. 85-106.

Malows, C. L. "Some Comments on Cp:" Technometrics (November 1973), pp. 661-75.

Pagano, Marcello, and Michael J. Hartley. "On Fitting Distributed Lag Models Subject to Polynomial Restrictions, "Nournal of Econometrics (June 1981), pp. 171-98.

Schwarz, Gideon. "Estimating the Dimension of a Model," The Annals of Statistics (March 1978), pp. 461-64.

Thornton, Daniel L, and Dallas S. Batten. "What Do Almon's Endpoint Constraints Constrain?" (Federal Reserve Bank of St. Louis, 1984; processed).

Trivedi, P. K, and A. R. Pagan. "Polynomal Distributed Lags: A Unified Treatment," Economic Studies Quarterly (April 1979), pp. $37-49$.

\section{Appendix: Model Selection Criteria}

The criteria employed here can be outlined within the framework of the following distributed lag model:

$$
\text { (1) } \underline{Y}=X \underline{\beta}+\underline{\varepsilon},
$$

where $X$ is a $T$ by $(Q+1)$ matrix of distributed lag variables, $\beta$ is a $(\ell+1)$ by 1 vector of parameters and $\varepsilon$ is a $T$ by 1 vector of disturbances. The initial step in implementing any of these techniques is to specify a maximum lag length, $\mathrm{L}$.

\section{Magano-Hardiey 1-test}

The Pagano-Hartley technique employs a GramSchmidt decomposition of the observation matrix. Specifically,

$$
\mathrm{X}=\mathrm{QN} \text {, }
$$

where $Q$ is a matrix whose columns form an ortho- normal basis for $\mathrm{X}$, and $\mathrm{N}$ is an upper triangular matrix with positive diagonal elements. Equation (1) can now be rewritten as

(2) $\underline{Y}=\mathrm{QN} \underline{\beta}+\underline{\varepsilon}=\mathrm{Q} \lambda+\underline{\varepsilon}$, where $\lambda=\mathrm{N} \underline{\beta}$.

To select the appropriate lag length, Pagano and Hartley suggest choosing the smallest $\mathbf{j}$ for which the hypothesis,

$$
\mathrm{H}_{\mathrm{L}_{2} \ldots ;}: \lambda_{\mathbf{1}, \ldots \mathrm{j}}=\mathbf{0},
$$

can be rejected. The $\mathrm{PH}$ technique also enables efficient calculation of the other lag-length selection statistics discussed below.

\section{Mallows' Cp-statistic}

An alternative to the PH technique is to consider minimizing some function of the residual sum of squares. One such statistic is Mallows' Cp-statistic, 
which is based upon a mean square error prediction norm. The Cp-statistic is defined as

$$
\begin{aligned}
C p_{L-i}=\frac{1}{s^{2}} \operatorname{RSS}_{L-i}-T+2(L+1-j), \\
\\
j=0,1, \ldots, L,
\end{aligned}
$$

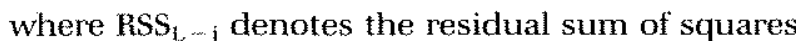
with j restrictions imposed and $s^{2}=\mathrm{RSS}_{1} / \mathrm{T}-\mathrm{L}-1$ ). As $\mathrm{j}$ increases from zero to $\mathrm{L}$, the Cp-statistic trades off some reduction in the variance of prediction for an increase in the bias. The value of $j$ for which the Cp-statistic is a minimum is the one that minimizes the expected mean square error of prediction. It can be shown that the Cp-statistic will attain a local minimum whenever the $\mathbf{t}$-statistic on the marginal distributed lag coefficient is greater than or equal to $\sqrt{2}$.

\section{Akaike's FPE Criterion}

Another criterion based on a mean square error prediction norm is Akaike's Final Prediction Error (FPE) criterion, defined as

$$
\mathrm{FPE}_{\mathrm{L},-\mathrm{i}}=\frac{T+(L+1-\mathrm{j})}{\mathrm{T}-(\mathrm{L}+1-\mathrm{j})} \cdot \frac{\mathrm{RSS}_{\mathrm{L}-\mathrm{i}}}{T}, \mathrm{j}=0,1, \ldots, L
$$

Like Mallows' Cp-statistic, the FPE criterion attempts to balance the "risk" due to bias when shorter lag lengths are selected against the "risk" due to the increase in variance when longer lag lengths are chosen. Hsiao (1981) has shown that minimizing the FPE is equivalent to applying an approximate sequential $F$-test with varying significance levels.

\section{Bayesian Criteria}

Two Bayesian criteria have been suggested that select the correct lag length asymptotically. The first of these is Schwarz' Bayesian Information Criterion (SBIC) and is given by

$$
\begin{aligned}
\mathrm{SBIC}=\ln \left(\frac{\mathrm{RSS}_{\mathrm{L}-\mathrm{j}}}{\mathrm{T}-\mathrm{L}-\mathbf{1}+\mathrm{j}}\right)+(\mathrm{L}+\mathbf{1}-\mathrm{j}) \frac{\ln \mathrm{T}}{\mathrm{T}}, \\
\mathrm{j}=0, \mathbf{1}, \ldots, \mathrm{L} .
\end{aligned}
$$

The second, suggested by Geweke and Meese, is the Bayesian Estimation Criterion (BEC) given by

$$
\begin{aligned}
\mathrm{BEC}=\frac{\mathrm{RSS}_{\mathrm{L}-\mathrm{i}}}{\mathrm{T}-\mathrm{L}-\mathbf{1}+\mathrm{j}}+(\mathrm{L}+1-\mathrm{j}) \mathrm{s}^{2}\left(\frac{\mathrm{In} \mathrm{T}}{\mathrm{T}-\mathrm{L}-1}\right) \\
j=0,1, \ldots, \mathrm{L} .
\end{aligned}
$$

Since choosing a lag length that is too long does not result in biased estimates of the distributed lag parameters, the only advantage of the Bayesian criteria is asymptotic efficiency.

\section{The Standard F-test}

A final procedure involves calculating a sequential F-statistic, defined as

$$
\left.F_{1,-i}=\left(\operatorname{RSS}_{1,-j-1}-\operatorname{RSS}_{1}\right) / \mathbf{j}+1\right) \mathrm{s}^{2}, \quad j=0,1, \ldots, L,
$$

and selecting the lag length as the first $\mathrm{L}-\mathrm{j}$ for which the null hypothesis, $\beta_{l}=\beta_{l_{1}-1}=\ldots=\beta_{L-i}$ $=0$, is rejected.

These procedures also can be applied to the problem of polynomial degree selection. Once the lag length is selected, determining the polynomial degree amounts to nothing more than selecting the length of the vector of polynomial coefficients. 